\title{
Zur Geschichte der Materialforschung
}

\author{
Klaus Hentschel und Carsten Reinhardt
}

Materialien sind gleichermaßen Rohstoffe und Erzeugnisse handwerklicher und industrieller Produktion, Gegenstände und Hilfsmittel der Forschung in naturwissenschaftlichen wie technischen Kontexten sowie Ausdrucksmittel und Träger kultureller Bedeutung. Ihre Erforschung ist sowohl Thema der Wissenschafts- und Technikgeschichte als auch der Alltags- und Kulturgeschichte. Im einleitenden Beitrag von Klaus Hentschel wird die kontroverse Frage untersucht, ob sich die aus den historischen Wurzeln der Metallurgie, Festkörperphysik und physikalischen Chemie sowie anderen Teilgebieten der Chemie wie etwa Polymer- und Keramikforschung entstandene Materialwissenschaft (engl. materials science) heute zu einer konvergenten Disziplin oder gar ,Superdisziplin' entwickelt hat, wie einige Materialforscher meinen, oder ob es sich nach wie vor um ein hybrides, lockeres und möglicherweise auch sozial instabiles Forschungsfeld handelt, wovon die auch im vorliegenden Themenheft von NTM vertretene Bernadette Bensaude-Vincent in einem Aufsatz von 2001 ausgeht. Neben den herkömmlichen Disziplingrenzen werden aber auch andere Fundamente des traditionellen Wissenschaftssystems von der materials science infrage gestellt, so etwa (i) die Unterscheidung zwischen Grundlagenforschung und angewandter Forschung (wo wäre etwa die aktuelle Suche nach hochtemperatursupraleitenden Keramiken einzuordnen?); (ii) die Unterscheidung zwischen Forschung und Entwicklung (wohin gehört etwa die Herstellung neuer Materialien mit ganz bestimmten gewünschten Stoffeigenschaften, wie sie in der heutigen Polymerchemie gang und gäbe ist?) und (iii) die Trennung universitärer von industrieller Forschung, da gerade die Materialforschung sich in komplexen Netzwerken öffentlicher und privater Forschungsträger abspielt, die je nach Arbeitsfeld lokalen, 
regionalen, nationalen oder internationalen Zuschnitt haben können (siehe dazu Luxbacher 2011 in diesem Heft). ${ }^{1}$

Erstaunlicherweise wird diese spannende Geschichte der Materialien und ihrer Erforschung von Wissenschafts- und Technikhistorikern erst seit kurzem intensiver bearbeitet. In diesem Kontext seien vor allem BensaudeVincent sowie Ursula Klein und Wolfgang Lefèvre genannt: Erstere hat für die USA, die in der Materialforschung insgesamt wohl federführend sind, die disziplinäre Genese der Materialforschung untersucht (Bensaude-Vincent 2001) und im Anschluss daran auch eine verdienstvolle Website kreiert, an der unter maßgeblicher Beteiligung von Arne Hessenbruch bis 2002 aktiv gearbeitet wurde. Leider scheinen diese historischen Ansätze einer internetbasierten Aufarbeitung des Feldes inzwischen wieder zum Erliegen gekommen zu sein. $^{2}$

Klein und Lefèvre dagegen nähern sich in ihren wissenschaftsgeschichtlichen Betrachtungen dem Thema aus einem ganz anderen Blickwinkel und zwar aus dem der historischen Ontologie (Klein/Lefèvre 2007). Während wir gemeinhin davon ausgehen, dass die Welt der Stoffe besonders stabil ist, haben sie, aber auch Ian Hacking (Hacking 2002) und andere anhand von historischen Fallbeispielen und durch wissenschaftstheoretische Analysen aufgezeigt, dass zumindest die Klassifikationssysteme, mit denen wir Stoffe ordnen und in Gruppen einteilen, historisch höchst variabel gewesen sind. So klassifizierte etwa Lavoisier, allgemein als Begründer der modernen Chemie gefeiert, Licht (lumière) und Wärmestoff (calorique) noch unter die Elementarstoffe seines neuen chemischen Systems der Elemente. Bei vielen weiteren Stoffen, an die frühere Generationen geglaubt haben, gehen wir mittlerweile nicht mehr davon aus, dass sie existieren, so zum Beispiel bei den Imponderabilien, das heißt, den nichtwägbaren Fluida des ,Standardmodells' des 18. Jahrhunderts (Heilbron 1993). Und auch die Grenzen zwischen den Stoffklassen haben sich permanent verschoben, ganz zu schweigen davon, dass ständig neue Materialien hinzugekommen sind.

Das Spannungsfeld zwischen institutioneller Entwicklung und epistemologischen Fragen wird im Rahmen dieses Sonderhefts von NTM thematisiert, während die eher auf soziologische Aspekte rekurrierenden Beiträge von Carsten Reinhardt und Joachim Schummer im nächsten Heft erscheinen werden. Im Mittelpunkt stehen die Geschichte chemischer und physikalischer Verfahren der Materialforschung, der Materialwissenschaften sowie neuere Ansätze der Wissenschaftsgeschichte der Stoffe. Dabei stehen die Beziehungen im Zentrum, die die entstehenden Materialwissenschaften zwischen solch klassischen Disziplinen wie Physik und Chemie geprägt haben. Insbesondere wird nach möglichen historiographischen Zugängen, der langfristigen Entwicklung der zugrundeliegenden Konzepte, der institutionellen Stellung in konkreten Konstellationen, der Rolle der Luftfahrtforschung und des Militärs (siehe hierzu Haka 2011 in diesem Heft), der habituell geprägten Identitäts- 
bildung durch Methodentransformationen sowie nach der Stellung zwischen Fachwissenschaft und Öffentlichkeit gefragt. Bei der Auswahl wurden ganz bewusst unterschiedliche historiographische Zugänge zum Thema gewählt, von fachnahen über technikhistorische bis hin zu sozialwissenschaftlichen und philosophischen Perspektiven. Die meisten der Beiträge gehen auf Vorträge zurück, die im Frühjahr 2009 auf der gemeinsamen Jahrestagung des Fachverbands Geschichte der Physik der Deutschen Physikalischen Gesellschaft und der Fachgruppe Geschichte der Chemie der Gesellschaft Deutscher Chemiker in Göttingen gehalten wurden (siehe dazu auch die Abstracts, online verfügbar unter http://www.dpg-physik.de/dpg/gliederung/fv/gp/pdf/ abstracts2009.pdf; letzter Zugriff: 26.11.2010).

\section{Anmerkungen}

1 Interessante Beispiele bieten etwa die Fallstudien von Arne Hessenbruch unter anderem zur Materialforschung in Dänemark, Griechenland, Portugal, Spanien im Vergleich mit den USA. Sie wurden über die Website der Société Chimique de France bis 2009 auch online verfügbar gemacht. Weitere Vergleiche beziehungsweise analoge Untersuchungen für andere wichtige Zentren der Materialforschung, wie etwa die Sowjetunion/Russland oder Deutschland, stehen noch aus.

2 Bis 2009 wurde diese Seite aktualisiert: http://authors.library.caltech.edu/5456/1/ hrst.mit.edu/hrs/materials/public/index.html. Einige Interviews der Gruppe um Bernadette Bensaude-Vincent finden sich nun auch unter http://www.sho.espci.fr/ (letzter Zugriff am 13.1.2011).

\section{Literatur}

Bensaude-Vincent, Bernadette, 2001. The Construction of a Discipline. Materials Science in the United States. Historical Studies in the Physical and Biological Sciences, 31, 223-248.

Hacking, Ian, 2002. Historical Ontology. Cambridge, MA: Harvard University Press.

Heilbron, John, 1993. Weighing Imponderables and other Quantitative Science around 1800. Berkeley [= Historical Studies in the Physical and Biological Sciences; 24, 1, Suppl.].

Klein, Ursula/Lefèvre, Wolfgang, 2007. Materials in Eighteenth-Century Science. A Historical Ontology. Cambridge, MA: MIT Press. 